\title{
PARACOCCIDIOIDOMICOSE GENITAL FEMININA DESCRIÇÃO DE UM CASO CLÍNICO
}

E. P. CaMPOS (1), L. N. TORChio (2), P. R. L. LMMa (3), F. C. B. Gones (3),

A. C. M. FERACIN (4) R. G. SCHFilliNi (5) e C. E. BACCHI (6)

\section{R E S U M O}

A paracoccidioidomicose é doença sistêmica que atinge mais o sexo masculino do que o feminino. Modelos experimentais mostram maior sensibilidade dos machos do que as fêmeas à dsseminação da doença. A literatura médica é rica em relatos de casos de Pbmicose do trato genital masculino. A raridade da Pbmicose sistêmica na mulher devido à ação hormonal tornou inusitada a descrição de um caso com envolvimneto genital. Paciente de 57 anos, branca, procedente de Conchas (SP), queixando-se de dor abdominal há 10 anos, difusa, seguida de cólica periumbelical periódica, com diarréia e obstipação alternadas. Piorou desse quadro e evoluiu para abdome agudo cirúrgico. $O$ anátomo patológico revelou Pbmicose no epiplon e ovário direito. A biópsia do endométrio mostrou endometrite crônica granulomatosa. A radiologia e a planigrafia revelaram nó. dulo parahilar direito. Os achados clínicos e laboratoriais permitiram explicar a provável origem do envolvimento genital.

UNITERMOS: Paracoccidioidomicose humana - Epiplon - Ovário - Endométrio.

\section{N T R O D U C A A O}

A paracoccidioidomicose, doença sistêmica descrita na América Tropical, é particularmente frequiente na região de Botucatu 4,11 . O fungo atingiria os pulmões pela via inalatória onde se estabeleceria o complexo primário semelhante ao da tuberculose, evoluindo ou não para a cura $2,10,13,15$.

A doença de Lutz atinge mais frequientemente o sexo masculino do que o feminino, segundo diversos Autores 2,4,6,13,15. Por outro lado, acomete ambos os sexos em igual proporção no período pré-puberal $9,13,14$.
Estudos experimentais em animais, evidenciaram maior sensibilidade dos machos à Pbmicose $5,9,12,14,18$.

A literatura médica é rica na descrição de casos de Pbmicose do trato genital masculino, porém, não se encontrou relato de pacientes com acometimento do aparelho genital feminino interno.

\section{DEScriçâo do caso clfnico}

M.I.F.C., internada no dia 03-05-84, 57 anos, feminina, branca, prendas domésticas, natural

(1) Professor Adjunto do Departamento de Moléstias Infecciosas e Parasitarias, Dermatologia e Radiologia

(2) Residente $1 .^{\circ}$ ano do Departamento de Moléstias Infecciosas e Parasitárias, Dermatologia e Radiologia

(3) Internos do 5.0 ano de Medicina

(4) Residente do $1 .^{\circ}$ ano do Departamento de Ginecologia e Obstetricia

(5) Auxiliar de Ensino - Disciplina de Radiologia

(6) Pós-graduando - Departamento de Patologia da F.M. 
CAMPOS. E. P.: TORCHIO, C. N.; LTMA, P. R. L.; GOMES, F. C. B.; FERACIN, A. C. M.: SCHEILINT, R. C. \& BACCHI, C. E. - Paracoccndioidomicose genital feminina. Descriçāo de um caso clínico. Rev. Inst. Med. trop. São Paalo 28: 56-60, 1986

de Porangaba e procedente de Conchas. S.P. Queixava-se de "dor de barriga" há 10 anos. de evolução insidiosa, difusa, continua, moderada, sem desencadeantes ou fatores de piora e melhora da dor, com hábito intestinal normal. Após alguns meses a dor mudou para cólica peri-umbelical mais intensa, que melhorava com o eliminar de flatus e antiespasmódicos, piorando à ingestão de alimentos gordurosos e diarréia alternada com obstipação intestinal de até 15 dias. Tratou de "colite" durante dez anos. Há três meses; calafrios e sudorese se associaram à piora da dor. Há dois meses, procurou médico que encontrou massa tumoral na região ovariana direita ao exame ginecológico e indicou laparatomia exploradora, que mostrou nódulos peritoneais, não caseosos e $500 \mathrm{ml}$ de líquido amarelo-citrino presentes na cavidade abdominal, Realizou ooforectomia à direita e biópsia de nódulo peritoneal, cujo diagnóstico anátomo-patológico foi de paracoccidioidomicose.

I.S.D.A., referia fraqueza e perda de 6 quilos nos últimos três meses. Negava qualquer alteração nos demais aparelhos ou sistemas.

Antecedentes pessoais: passado pulmonar ausente e não referia contacto com aves e pombos. Morou em zona rural, onde havia lavoura de arroz, feijăo e milho. Possuia hábito de mascar capim e não trabalhou na lavoura. Não sou. be referir data de início da menopausa.

Exame físico geral: paciente em bom estado geral; eupnéica, acianótica, anictérica, afebril, hidratada, ativa e contatuando bem. Peso = $52 \mathrm{~kg}$; Altura $=1,52 \mathrm{~m} ; P=F C=76 \mathrm{bpm}$ $\mathrm{FR}:=20 \mathrm{mpm} ; \mathrm{T}=36,5^{\circ} \mathrm{C} ; \mathrm{PA}=14 \times 8 \mathrm{mmHg}$; brevilínea.

Pele: cicatriz infraumbelical. demais nada digno de nota, mucosas úmidas e descoradas $(+/++)$; gânglios; nada digno de nota, T.C.S.C.: sem edema, sem enfisema e distribuição regular. Musculatura normo-trófica, ossos sem deformidade.

Exame físico especial: cabeça nada digno de nota, prótese dentária. Pescoço normal; tireóide normal à palpação.

Pré-córdio: S.S. Mesocardico $(+)$, suave. Pulmões: semiologia normal. Abdome: cicatriz mediana infraumbelical de $15 \mathrm{~cm}$ à inspeção.
Palpação superficial: nada digno de nota. Figado percutido e palpado com características normais, baço nảo palpável e năo percutível. Percussão: nada digno de nota e ruidos hidro aéreos presentes e norma1s. Giordano negativo Membros e articulaçôes, nada digno de nota. Neurológico: fácies; atitude, reflexos, sensibilidade e motricidade normais. Avaliação O.R. Laringológica: normal. Exame Ginecológico: presença de cicatriz mediana infra-umbelical. Genitália externa compatível com a idade. Genitália interna-espéculo vaginal: paredes atrofjcas, pregueamento diminuído sem secreçāo em fundo de saco. Colo pequeno e orifício externo em fenda transversa. Schiller: nada digno de nota. Toque: vagina pérvea, elasticidade dimimuida, profundidade $7 \mathrm{~cm}$, sem ondulação, colo apagado, pequeno, de consistência cartilaginosa no eixo vaginal. Útero: retrovertido fixo. Ausência de nodulações em anexos. Histerometria: $8 \mathrm{~cm}$.

Hipótese diagnóstica: Pbmicose de ovário e peritoneo intestinal.

Exames complementares realizados antes $e$ durante a internação revelaram: Hemograma hematócrito $=31 \%$; V.H.S. $=11 \mathrm{~cm}-1 .{ }^{\mathrm{a}}$ hora; $\mathrm{GB}=3.000 / \mathrm{mm}^{3} ;$ segmentados $=57 \%$; baso filo $=1 \%$; eosinófilo $=2 \%$; linfócitos $=32 \%$; monócitos $=8 \%$; plaquetas normais; hipocromia $=(++)$ e ponteados basófilos.

Avaliação bioquímica mostrou glicemia de jejum $=84 \mathrm{mg} \% ; \mathrm{Na}+=144 \mathrm{mEq} / 1 ; \mathrm{K}+=$ $4,1 \mathrm{mEq} / 1 ; \mathrm{Ca}++=9,0 \mathrm{mg} \% ;$ T.G.O. $=24 \mathrm{UI}$ e TGP $=43$ UI. Eletroforese de proteinas: albumina $=4,55 \mathrm{~g} \% ;$ alfa $_{1}=0,27 \mathrm{~g} \% ;$ alfa $_{2}=$ $0,38 \mathrm{~g} \%$; Beta $=0,66 \mathrm{~g} \%$ e gama $=1,04 \mathrm{~g} \%$ e relaçāo $A / G=1,94$.

Dosagem sérica hormonal por radioimunoensaio revelou: F.S.H. $=161 ; 3 \mathrm{ng} / \mathrm{dl} ; \mathrm{L} . \mathrm{H} .=$ $41,3 \mathrm{ng} / \mathrm{dl}$; P.R.L. menor do que $2,9 \mathrm{ng} / \mathrm{dl}$; testosterona $=49 \mathrm{ng} / \mathrm{dl}$ e estradiol $=2,5 \mathrm{nd} / \mathrm{dr}$; compativeis com a menopausa.

A função supra-renal mostrou-se normal ao teste de cortisol sérico.

Exame de urina: $p H=6,5$; glicosúria, cetonúria e proteinuúria ausentes. Sedimento 8 a 9 leucócitos por campo; raras células de descamação e 5 a 6 hemácias por campo. Parasitológico de fezes completo: negativo. 
CAMPOS, E. P, TORCHIO; L. N ; LIMA, P R L: COMES, P C B ; FERACIN, A C. M.; SCHELLINI, R C \& BACCHI, C. E. - Paracoceidiaidomiense genital feminina Descriçlo de um caso clinico Rev. Inst. Med trop. Säo Paulo as: 5660,1986

Pesquisas de B.A.R. e fundos no escarro: negativas: Paracoccidioidina: duas vezes, negativa.

Estudo radiológico revelou trânsito intestinal normal; torácico-nódulo hilar direito, demais nada digno de nota e a planigrafia confirmou nódulo hilar com discreta compressâo do brônquio direito.

A ultrassonografia nada revelou. A sorologia mostrou precipitina $(+)$, fixação do complemento (Ag polissacarideo), negativa, fixaçào do complemento (filtrado de cultura) - negativo, imunofluorescència indireta, 1/32 e imunodifusâo em gel, negativa denotando ausência de atividade da doença.

Histopatológico: as biópsias de epiplon, trompa-uterina e de ovário evidenciaram processo inflamatório crônico granulomatoso năo caseoso com raras formas de $\mathbf{P}$. brasiliensis (Fig, 1), Biópsia de endométrio: endometrite crônica granulomatosa (pesquisa de B.A.A.R. e fungos negativas).

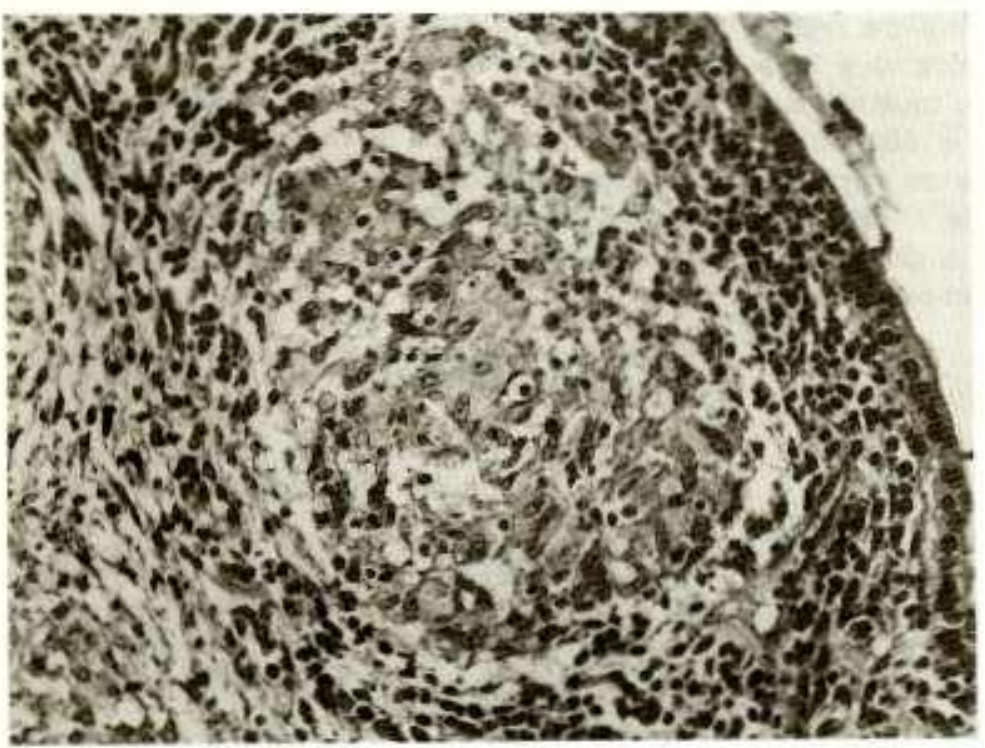

Fig 1 - Trompa aterias com reaço inflamatórin granulomatosa no obrio. Hematoxilina e Eosina $160 \times$

\section{COMENTARIOS}

O abdome agudo apresentado pela pacience seria explicado pela peritonite granuiomatosa evidenciada à laparoscopia. A adenopatia perito. neo-intestinal seria proveniente da disseminação linfohematogênica fơco pulmonar 2,7,5,10.13,15,17 ou eventualmente a partir de foco primário quiescente intestinal $2,8,13,17$. A peritonite seria desencadeada provavelmente. por dois mecanis. mos análogos aos da tuberculose: via trompa de Falópio ou via linfonodes mesentéricos $23,13,17$. Disseminaçăo hemolinfática atual ficaria excluída pela ausência da doença pulmonar ativa análo. go ao que se observaria na tuberculose 10.13.15.17.

A Pbmicose genital masculina resultaria da disseminaçăo fúngica pela via hemolinfática $1,3,6,16$. A lesăo genital de modo geral, acompanharia peneumopatia ativa da Pbmicose 1,3,6,26.
A evidente suscetibilidade do sexo masculino a micose explicaria o maior numero de ca. sos de Pbmicose sistêmica, bem como o encontro de doentes com envolvimento genital 1,3,6,16,16.

A Pbmicose atingiria, mais frequentemente, o epididimo e o testículo seguidos em ordem decrescente da próstata, ureter e pênis 1,3,6,16.

Experimentos realizados por alguns Autores, em animais machos e fêmeas, evidenciaram maior crescimento de colônias fúngicas nas culturas de fragmentos de órgâos oriundos dos machos 5,9,12,13. Fraçōes de tecidos provenientes de machos adultos e pré-púberes demonstraram, pós-cultivo, maior número de colonias fúngicas nestes do que naqueles, sugerindo a influência do hormônio masculino na origem do processo $5,9,12,14,18$, Sabe-se que a proporção de doen- 
CAMPOS, E. P.; TORCHIO, L. N. LIMA, P. R. C.: GOMES, F. C. B.; FERACIN, A. C. M.; SCHELLINI, R. C. \& BACCHI, C. E. - Paracoccidjoidomicose genital feminina. Dascrição de um caso clinico. Rev. Inst. Med. trop. São Paalo \&8: 56-60, 1986.

tes com Pbmicose é semelhante para os sexos no periodo pré-puberal $9,13,14$.

A estimulação imunitária oriunda da presença do hormônio feminino, seria fundamental na proteção da doença blastomicótica $9,12,13,14$. $O$ estrógeno estimularia as atividades linfocítica e macrofágica, propiciando melhor capacidade de defesa à doença, segundo demonstraram alguns experimentos "in vitro" $9,12,13$.

No presente caso encontrou se processo granulomatoso no peritoneo, no ovário direito $\mathrm{e}$ no endométrio. A explicação para o envolvimento desses órgãos obedeceria critério análo go ao descrito na tuberculose $6,7,8,10,13,15,17$.

A radiologia e a planigrafria pulmonares possibilitaram compreender a provável origem do comprometimento genital da Pbmicose. $O$ nódulo pulmonar hilar direito sugeriu-nos complexo residual simile ao de Ghon, que explicaria o envolvimento do órgão genital feminino "a posteriori". A função adrenal normal excluiu possivel acometimento precoce e/ou tardio dessa glândula pela doença paracoccidioidomicótica crônica ativa.

O aparecimento da doença durante o climatério permitiu supor possivel reativação endógena de foco abdominal, pelas modificaçōes hormonais descritas e pela ausência de atividade da moléstia demonstrada ao exame sorológico dessa paciente.

\section{SUMMARY}

\section{Paracoccidioidomycosis brasiliensis. Case report of female genital involvement}

Paracoccidioidomycosis is a systemic dìsease that predominantly affects males. Experimental models suggest that males are more susceptible to the disseminated form of the disease.

In vitro siudies have documented growth inhibition in the presence of estradiol and progesterone whereas testosterone does not.

Paracoccidioidomycosis has been well described involving the male genitalia. Female genital involvement is rare; perhaps related to hormonal inhibition of fungal growth.
A 57 year-old causasian female is described who presented with a ten year history of diffuse abdominal pain with alternating diarrhea and constipation. She went on to present with an acute surgical abdomen and proceeded to la parotomy.

Histopathological study revealed involvement of the omentum, fallopian tubes and right ovary with Paracoccidioidomycosis. Endometrial biopsy revealed chronic granulomatous endometritis, however, no fungus was visualized. Chest $\mathrm{X}$ ray and tomography were consistent with right parahilar involvement. We suggest, pathophysiologically, that the genital disease represents reactivation of an abdominal focus.

\section{REERENGIAS BIBLIOGRAFICAS}

i. BILIIS, A. \& SILVEIRA, E. - Blastomicose sul ame ricana do ureter. Apresentação de um-caso. Rev. Ass. Med. bras, 19: 463.466, 1973

2. BRASS, KC. - Observaciones sobre 1a anatomia pato logica, patogenesis $y$ evolucion de la paracoccidioidomicosis. Mycopatholgis (Den Haag) 37: 119-138, 1969.

3. BRITO, R. R. \& CAPRINI, N. - Blastomicose ds próstata. Rev, paul. Med, 54: 112-116, 1959.

4: CAMPOS, E. P.; SARTOR, J. M.; HETCH, M. L. \& FRANCO, M. F. - Aspectos clínicos e evolutivos do 47 doentes com paracoccidiotdomicose tratados pela Anfotericina B. Rev. Inst. Med. trop. Säo Paulo 26: 212-217, 1984

5. CAMPOS, E. P.; TANI, E. \& MACKENZIE, D. W, R. - Estudo do comportamento da infecção pelo Cryptococcus 3166 N.C.P.F. em ratos machos. XXI Congresso Soc. Bras. Pnewmol., 21 a 25 novembro/1982 p. 209 (Supl.), Vol. 8 J. Pneumol:

6. CECHELIA, M. S.; MELO, C. R.; MELU, I. S.; LONDERO, A. T.; BARRETO, S. M. \& GAIGER, A. M. - Paracoccidioidomicose genital masculino. Rev. Inst. Med, trop. São Paula 25: 240-245, 1982.

7. FIAEHO, A. S. - Localizações pulmonares da "Milcose de Lutz". Anatomia patológica e patogenia. Importância de seu estudo na patologia pulmonar. [Tese]. Rio de alneiro, 1946.

B. FIORILLO, A. M; MARTINEZ, R. \& DE MORAES, C. R. - Lesర̃es do aparelho digestivo. In: Paracoccidlol. domicose. Del Negro, G; Lacaz, C. S. \& Fiorllla, A. M. - (1.a ed.). São Paulo, Sarvier, Cap. 18, 179-193, 1982.

9. GIRALDO, R. R. - Experimental paracoceidfoidomycosis in mice. Influence of sex on the infection. [Dissertation for the Master's Degree in Clinical Medicine]. London School Hyglene \& Tropical Mediicne, 1975. 
CAMPOS, E. P.; TORCHIO, L. N.; LIMA, P. R. L.; GOMES, F. C. B.; FERACIN, A. C. M.; SCHELLINI, R. C. \& BACCHI, C. E. - Paracoccidioidomicose genital feminira. Descriçâo đe um caso clinico. Rev. Inst. Med. trop. São Paulo 28: 56-60, 1986.

10. MACKINNON, J. E. - Pathogenesis of South American Blastomycosis. Trans. Roy. Soc. trop. Med. Hyg. 53: 487.494, 1959.

11. MARQUES, S. A.; FRANCO, M. F.; MENDES, R. P.: SILVA, N. L. A.; BACCILI, C.; CURCELLI, E. D.; FERACIN, A. C. M.; OLIVEIRA, C. S.; FAGLIARINI, J. V. \& DILION, N. A. - Aspectos epidemiológicos da paracoccidioldomicose na área endêmics de Botucatu (Săo Paulo - Brasil). Rev. Inst. Med. trop. Sāo Paulo 25: 87-92, 1983.

12. MUCHMORE, H. G.; McKOWN, B. A. \& MOHR, J. A. - Efectos de las hormonas esteroides sobre la proliferacion de Paracoccldioides brasiliensis. Bol. Ofic. Sanit. Panamer. 77: 55-60, 1974.

13. RESTREPO, A. M. - Atualizaciones sobre paracoccldioidomicose. Acta Med. Colombiana 3: 33-65, 1978.

14. SCHELEGEL, R. J. \& BELLANTI, J. A. - Increased susceptibility of males to infection. Lancet 12: 826827, 1969.
15. SEVERO, L. C. - Paracoccidioldomicose. Estudo clínico e parasitológico das lesões pulmonares e seu diagnóstico. [Tese - Mestrado]. Universidade Federal do Rio Grande do SuI, Porto Alegre, 1979.

16. SILVEIRA, E.; BILlIS, A.; REVISAN, M. A. S. \& VIEIRA, R. J. - South American Blastomycosis of the ureter. Am. J. trop. Med. Hyg. 25: 530-531, 1976.

17. STEAD, W. W. \& BATES, J. H. - Tuberculosis in Harrison's principle of internal Medicine, Petersdorf, R. G.; Adams, R. D.; Braunwald, E.; Isselbacher, K. J.; Martin, J. B. \& Wilson, J. D. - (10.ed.). New York, McGraw-Hill, Cap. 174, 1019-1030, 1983.

18. WALShBURN, T. C.; MEDEARIS JR., D. V. \& CHID. B. - Sex differences in susceptibility to infections. Pediatrics 35: 57.64, 1965.

Recebido para publicação : em 14/12/1984. 